09.1;01.1

\title{
Моделирование оптимальной оптической системы ввода/вывода излучения для реализации эффективного зондового усиления электромагнитного поля для случая непрозрачных образцов
}

\author{
(C) М.А. Трусов ${ }^{1}$, А.Е. Ефимов ${ }^{2}$, Д.О. Соловьева ${ }^{3}$, И.С. Васкан ${ }^{3,4}$, В.А. Олейников ${ }^{3}$, К.Е. Мочалов ${ }^{3, \uparrow}$ \\ ${ }^{1}$ Институт теоретической и экспериментальной фризики им. А.И. Алиханова Национального исследовательского центра \\ „Курчатовский институт“, Москва, Россия \\ 2 Национальный медицинский исследовательский центр трансплантологии и искусственных органов \\ им. акад. В.И. Шумакова Минздрава России, Москва, Россия \\ ${ }^{3}$ Институт биоорганической химии им. академиков М.М. Шемякина и Ю.А. Овчинникова РАН, Москва, Россия \\ ${ }^{4}$ Московский фризико-технический институт (Национальный исследовательский университет), Долгопрудный, Московская \\ обл., Россия \\ ฯ E-mail: mochalov@mail.ru
}

Поступило в Редакцию 9 октября 2020 г.

В окончательной редакции 23 ноября 2020 r.

Принято к публикации 27 ноября 2020 г.

\begin{abstract}
Рассмотрены конкурентные схемы построения оптической системы для комбинации сканирующего зондового микроскопа и оптического микроспектрометра, позволяющие исследовать непрозрачные образцы методом зондового усиления интенсивности комбинационного рассеяния. Произведен подбор оптимальных объективов для реализации каждой схемы с учетом наличия зонда сканирующего микроскопа. Количественно оценена эффективность применения каждой оптической схемы как для возбуждения сигнала комбинационного рассеяния, так и для сбора вторичного излучения. В результате выявлена наиболее эффективная с точки зрения параметра „возбуждение/сбор“ оптическая система.
\end{abstract}

Ключевые слова: сканирующая зондовая микроскопия, микроспектроскопия, зондовое усиление интенсивности комбинационного рассеяния.

DOI: 10.21883/PJTF.2021.05.50677.18576

В последнее десятилетие широкое распространение получили корреляционные методы микроскопических исследований, когда образец изучается несколькими комплементарными методами [1-5]. В связи с этим большой интерес представляет комбинация сканирующей зондовой микроскопии $(\mathrm{C} 3 \mathrm{M})$ со спектроскопией комбинационного рассеяния, ставшая основой метода локального зондового усиления интенсивности комбинационного рассеяния (tip enhanced Raman scattering, TERS) [6], в частности реализованная в уникальной научной установке - системе зондово-оптической 3D-корреляционной микроскопии, созданной авторами настоящей работы (http://ckp-rf.ru/usu/486825/). Благодаря возможности наномасштабной характеризации образцов метод TERS применяется как для химической визуализации различных материалов [7], так и для структурно-функциональных исследований биологических макромолекул [8]. На данный момент наилучшие результаты получены с использованием инвертированной оптической схемы микроспектрометра, позволяющей применять объективы с $N A>1$ [7,9]. Однако на практике зачастую приходится иметь дело с непрозрачными образцами, не позволяющими реализовать эту схему. Необходимость установки СЗМ-зонда в область между объективом и образцом значительно снижает числовую апертуру используемого объектива. Это в свою оче- редь снижает эффективность возбуждения $z$-компоненты электромагнитного поля, необходимой для усиления поля под зондом и сбора вторичного излучения. На данный момент существует ряд конкурентных технических решений, направленных на преодоление указанной проблемы, и целью настоящей работы являются их сравнение и выбор наиболее оптимальной оптической системы ввода/вывода излучения.

В общем случае для описания явления комбинационного рассеяния света используется дипольное приближение. На рисунке представлены общая схема расположения основных элементов при проведении TERS-экспериментов и диаграмма направленности распространяющегося излучения. В дипольном приближении относительная интенсивность излучения в элемент телесного угла, нормированная на единицу, дается известным выражением

$$
I=\frac{3}{8 \pi} \int_{\theta_{\min }}^{\theta_{\max }} d \theta \sin ^{3} \theta \int_{\varphi_{\min }}^{\varphi_{\max }} d \varphi,
$$

где $\theta$ и $\varphi-$ полярный и азимутальный углы соответственно. Для простоты примем, что ось диполя (ось $Z$ ) в данном случае ориентирована по нормали к образцу. Для достижения заметного усиления сигнала комбинационного рассеяния из-под зонда необходимо 


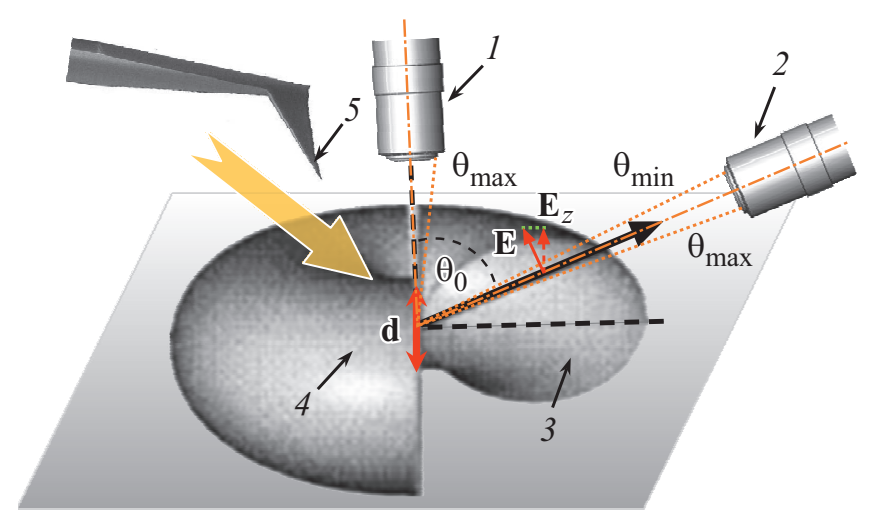

Схема расположения основных элементов при проведении традиционных TERS-экспериментов. 1 - объектив в позиции $1 ; 2$ - объектив в позиции $2 ; 3$ - электромагнитное поле, излучаемое диполем; 4 - излучающий диполь; 5 TERS-зонд.

обеспечить наличие вертикальной $(z)$ компоненты электромагнитного поля $\mathbf{E}$ в области острия зонда. Известно два основных варианта реализации данного условия. Первый вариант заключается в использовании верхнего объектива (объектив в позиции 1 на рисунке) и секторальной фазовращательной пластинки, разворачивающей плоскость поляризации в каждом секторе так, чтобы вектор электрического поля был направлен от оптической оси. В такой схеме при полной равномерной засветке входного кольцевого зрачка мы получим относительное значение интенсивности $z$-компоненты в падающей волне, равной

$$
\frac{\int_{\theta_{\min }}^{\theta_{\max }}(\tan \theta)^{3} d \theta}{\int_{\theta_{\min }}^{\theta_{\max }} \frac{\tan \theta}{(\cos \theta)^{2}} d \theta}=1-\frac{2 \ln \frac{\cos \theta_{\min }}{\cos \theta_{\max }}}{\left(\tan \theta_{\max }\right)^{2}-\left(\tan \theta_{\min }\right)^{2}},
$$

где $\theta_{\max }$ и $\theta_{\min }$ - максимальный и минимальный углы засветки зрачка объектива из точки фокуса.

Для более аккуратного математического описания данной оптической схемы необходимо также учесть фактор виньетирования (частичного затенения) фокусируемого пучка балкой СЗМ-зонда (кантилевера). Для этого достаточно ограничить пределы интегрирования по полярному углу в формуле (1) исходя из угла сходимости балки. Данный угол составляет примерно $30^{\circ}$ для промышленно изготавливаемых TERS-зондов. Следовательно, пределы интегрирования составляют $15<\theta<345^{\circ}$.

В частности, для зеркального объектива схемы Шмидта Thorlabs $40 \times / N A=0.50$ (Thorlabs Inc., США) с затененной центральной частью имеем $\theta_{\max }=30^{\circ}, \theta_{\min } \approx 14^{\circ}$, что дает относительное значение $z$-компоненты примерно 15\%. Для линзового объектива Mitutoyo Plan Apo HR Infinity Corrected Objective $50 \times / N A=0.75$ (Mitutoyo Corp., Япония) имеем $\theta_{\max }=30^{\circ}, \theta_{\min }=0^{\circ}$, что дает относительное значение $z$-компоненты примерно $32 \%$.
Использование двух типов объективов диктуется различием в их конструкции. Зеркальные объективы позволяют завести бо́льшую часть излучения в обход балки кантилевера, тогда как линзовые объективы имеют бо́льшую числовую апертуру.

Второй вариант заключается в использовании бокового объектива (объектив в позиции 2 на рисунке) типа Mitutoyo Plan Apo SL200 (Mitutoyo Corp., Япония), в который заводится линейно поляризованное лазерное излучение $p$-типа. При угле $40^{\circ}$ оптической оси объектива к вертикали (являющемся технически предельным) относительная величина интенсивности $z$-компоненты в падающей волне с таким объективом составит

$$
\left(\sin 40^{\circ}\right)^{2} \approx 41 \%
$$

Теперь приступим к анализу эффективности сбора вторичного излучения теми же объективами, которые были проанализированы выше. Вернемся к формуле (1) и данным рисунка. Классический вариант сбора „сверху“ (позиция 1 на рисунке) линзовым объективом с числовой апертурой NA с оптической осью, ориентированной вдоль оси диполя, обеспечивает следующую эффективность:

$$
\begin{aligned}
\eta & =\frac{3}{4} \int_{0}^{\arcsin N A} d \theta \sin ^{3} \theta=\frac{1}{2}-\frac{3}{4} \cos \arcsin N A \\
& +\frac{1}{4} \cos ^{3} \arcsin N A .
\end{aligned}
$$

Для объектива Mitutoyo $50 \times / N A=0.75$ эффективность сбора составляет примерно 7\%. Если использовать в такой же позиции зеркальный объектив схемы Шмидта, то формулу расчета эффективности нужно модифицировать

$$
\eta=\frac{3}{4} \int_{\theta_{\min }}^{\theta_{\max }} d \theta \sin ^{3} \theta
$$

где $\theta_{\max }$ определяется апертурой, а $\theta_{\min }$ - относительной величиной $r$ затененной области зрачка объектива. Из геометрических соображений легко вычислить, что

$$
\tan \theta_{\min }=\sqrt{\frac{r}{1+r}} \tan \theta_{\max } .
$$

Для зеркального объектива Thorlabs $40 \times / N A=0.50$ находим, что $\theta_{\max }=30^{\circ}, \theta_{\min } \approx 14^{\circ}$, и эффективность сбора составляет всего $\eta \approx 1.1 \%$.

Рассмотрим теперь вариант сбора „сбоку“ (позиция 2 на рисунке) линзовым объективом с оптической осью, наклоненной к вертикали на определенный угол $\theta_{0}$. Интегрирование по азимутальному углу в исходной формуле можно выполнить явно, и мы получаем следующее 
выражение:

$$
\begin{aligned}
& \eta=\frac{3}{8 \pi} \int_{\theta_{0}-\arcsin N A}^{\theta_{0}+\arcsin N A} 2 \sin ^{3} \theta \\
& \times \arccos \frac{\sqrt{1-N A^{2}}-\cos \theta_{0} \cos \theta}{\sin \theta_{0} \sin \theta} d \theta .
\end{aligned}
$$

Для варианта Mitutoyo Plan Apo SL200 $(N A=0.62)$ имеем $\arcsin N A \approx 38^{\circ}$. Рабочее расстояние объектива составляет $13 \mathrm{~mm}$, его диаметр $32 \mathrm{~mm}$, что и определяет упомянутый ранее его предельный наклон к вертикальной оси $\theta_{0} \approx 40^{\circ}$. При данных параметрах получаем величину эффективности сбора $\eta \approx 5.1 \%$.

Общую эффективности работы оптической системы можно представить как произведение эффективности возбуждения на эффективность сбора при фиксированной мощности возбуждающего излучения. Общая эффективность рассмотренных оптических схем реализации методики TERS выглядит следующим образом:

- для высокоапертурного объектива Mitutoyo $50 \times / N A=0.75$ в схеме „сверху“ $\sim 2.2 \%$;

- для зеркального объектива схемы Шмидта Thorlabs $40 \times / N A=0.50$ в схеме „сверху“ $\sim 0.17 \%$;

- для объектива Mitutoyo Plan Apo SL200 под углом $40^{\circ}$ к нормали $\sim 2.1 \%$.

Таким образом, мы установили, что для реализации методики TERS одинаково возможно использование как бокового объектива, установленного под углом, так и высокоапертурного объектива „сверху“. Важно отметить, что первый вариант значительно проще в реализации, однако в ряде случаев, например для недавно разработанных установок 3D-TERS [10], использование бокового объектива невозможно по конструктивным соображениям. Кроме того, боковой объектив в отличие от установленного сверху не позволяет реализовать методики стандартной широкопольной и конфокальной микроспектроскопии.

\section{Финансирование работы}

Работа выполнена при поддержке Российского научного фонда (проект № 18-19-00718).

\section{Конфликт интересов}

Авторы заявляют, что у них нет конфликта интересов.

\section{Список литературы}

[1] B. Joosten, M. Willemse, J. Fransen, A. Cambi, K. van den Dries, Front. Immunol., 9, 1908 (2018). DOI: $10.3389 /$ fimmu.2018.01908

[2] T.L. Burnett, P.J. Withers, Nature Mater., 18, 1041 (2019). DOI: $10.1038 / \mathrm{s} 41563-019-0402-8$
[3] A. Burel, M.-T. Lavault, C. Chevalier, H. Gnaegi, S. Prigent, A. Mucciolo, S.Dutertre, B.M. Humbel, T. Guillaudeux, I. Kolotuev, Development, 145 (12), dev160879 (2018). DOI: $10.1242 /$ dev.160879

[4] A.E. Efimov, I.I. Agapov, O.I. Agapova, V.A. Oleinikov, A.V. Mezin, M. Molinari, I. Nabiev, K.E. Mochalov, Rev. Sci. Instrum., 88 (2), 023701 (2017). DOI: 10.1063/1.4975202

[5] K.E. Mochalov, A.E. Efimov, A. Bobrovsky, I.I. Agapov, A.A. Chistyakov, V. Oleinikov, A. Sukhanova, I. Nabiev, ACS Nano, 7 (10), 8953 (2013). DOI: 10.1021/nn403448p

[6] J. Wessel, J. Opt. Soc. Am. B, 2 (9), 1538 (1985). DOI: $10.1364 / J O S A B .2 .001538$

[7] N. Kumar, B.M. Weckhuysen, A.J. Wain, A.J. Pollard, Nature Protocols, 14 (4), 1169 (2019). DOI: $10.1038 / \mathrm{s} 41596-019-0132-\mathrm{z}$

[8] C. D'Andrea, A. Foti, M. Cottat, M. Banchelli, C. Capitini, F. Barreca, C.Canale, M. de Angelis, A. Relini, O.M. Maragó, R. Pini, F. Chiti, P.G. Gucciardi, P. Matteini, Small, 14 (36), 1800890 (2018). DOI: 10.1002/smll.201800890

[9] L. Gao, H. Zhao, T. Li, P. Huo, D. Chen, B. Liu, Int. J. Mol. Sci., 19 (4), 1193 (2018). DOI: 10.3390/ijms 19041193

[10] K.E. Mochalov, A.A. Chistyakov, D.O. Solovyeva, A.V. Mezin, V.A. Oleinikov, I.S. Vaskan, M. Molinari, I.I. Agapov, I. Nabiev, A.E. Efimov, Ultramicroscopy, 182, 118 (2017). DOI: $10.1016 /$ j.ultramic.2017.06.022 